\title{
Some preliminary reflections on the epistemology of Computational Theory of Perceptions
}

\author{
Clara Barroso $^{1}$ Gracian Trivino $^{2}$ \\ ${ }^{1}$ Universidad de la Laguna, Canary Islands, Spain, Email:cbarroso@ull.es \\ ${ }^{2}$ European Centre for Soft Computing, Asturias, Spain, Email: gracian.trivino@softcomputing.es
}

\begin{abstract}
The impressive practical applications is one of the main reasons of success of Fuzzy Logic. The Computational Theory of Perceptions (CTP) is an extension of this theory that has not yet reached the same level of applicability.

We believe that a review of the fundamentals of CTP from a epistemological perspective would provide useful insights to contribute to energize its development.

This is a very preliminary paper where we make some reflections on epistemological aspects of CTP. We have used three representative Zadeh's papers to extract a set of relevant sentences describing CTP. Then, we analyzed this corpus of information from various epistemological points of view to obtain some provisional conclusions.
\end{abstract}

Keywords: Computational Theory of Perceptions, Epistemology

\section{Introduction}

According to Zadeh, Computational Theory of Perceptions (CTP) is an extension of Fuzzy Logic inspired by the remarkable human capacity to perform a wide variety of physical and mental tasks using fuzzy perceptions. Hopefully, this theory could open the way of new practical applications with comparable success to Fuzzy Logic. We think that a clarification on the fundamentals of CTP could be in order to contribute to its development.

As far as we know, there are not antecedents of an epistemological analysis applied to CTP.

The term theory, as the majority of words, is used in everyday natural language with different meanings depending on each situation type. The term perception has different meanings in Psychology and Philosophy. The concept of computational perception is not yet clearly defined. From a formal point of view, Epistemology provides mechanisms to analyze the available information about CTP. The aim of this paper is to explore the possibilities of applying here this type of analysis.

The input data to this preliminary analysis is a set of three representative papers by Lotfi Zadeh, namely, [1], [2] and [3].

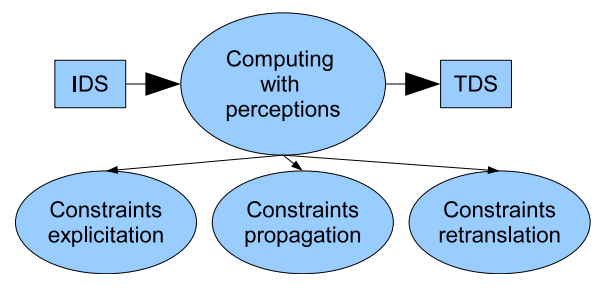

Figure 1: Conceptual diagram of computing with words and perceptions

First, we create an initial draft description of CTP consisting of a set of statements extracted from the analyzed papers. Then, we study this information using three epistemological perspectives.

In section 3 , we present some reflections about what is CTP from the perspective of the Logic of Science by K.R. Popper. In section 4, we present some reflections about who uses CTP from the perspective of the Disciplinary Matrix by T. S. Kuhn. Section 5 explore why to use CTP from the perspective of a rational knowledge of normative type.

In section 6 , we present a set of additional definitions that we introduced during our research on applying CTP in practice.

\section{Computational Theory of Perceptions}

CTP was introduced in the Zadeh's seminal paper "From computing with numbers to computing with words - from manipulation of measurements to manipulation of perceptions" [1] and further developed in subsequent papers, e.g., [2] [3] [4]. It is inspired by the human capability to perform tasks without using crisp measurements and numerical computations, e.g., driving in heavy traffic and playing golf. Underlying this capability is the brain's ability to manipulate perceptions. Moreover, this capability plays a key role in human recognition, decision and execution processes.

CTP provides a framework to develop computational systems with the capacity of computing with the meaning of natural language (NL) expressions, i.e., with the capacity of computing with imprecise descriptions of the world in a similar way that humans do it.

According to CTP, our perception of world is 


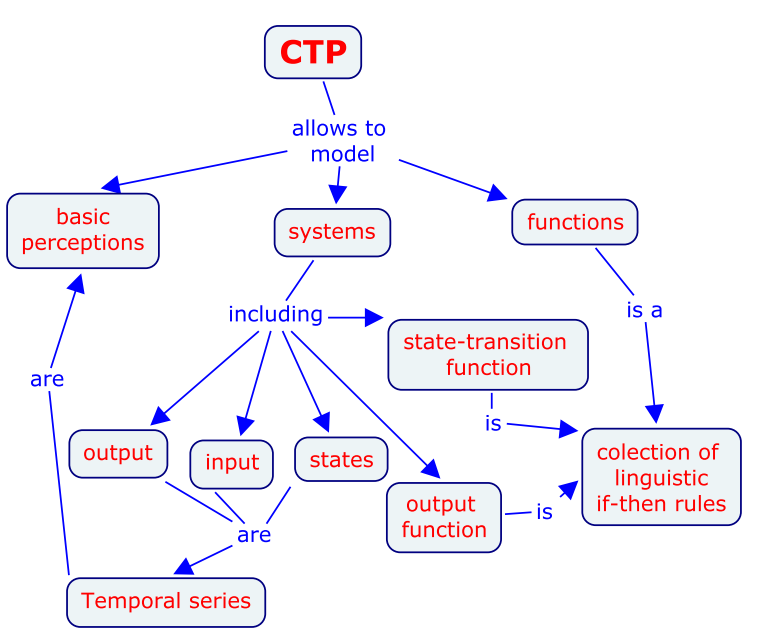

Figure 2: Concept Map of CTP

granular. A granule is a clump of elements which are drawn together by indistinguishability, similarity, proximity or functionality [5] [3]. The boundary of granules is fuzzy. Fuzziness of granules allow us to model the way in which human concepts are formed, organized and manipulated in an environment of imprecision, uncertainty and partial truth [6].

A granule underlies the concept of a linguistic variable [4]. A linguistic variable is a variable whose values are words or sentences in NL [7].

Figure 1 represents the basic conceptual structure of a system for computing with perceptions. The Initial Data Set (IDS) is a set of perceptions represented by propositions expressed in a subset of NL called Precisiated Natural Language (PNL). They contain the available information to answer a question of interest for the user of the computational system. The Terminal Data Set (TDS) is a perception (represented by a PNL proposition) corresponding with the answer to the question. The process module contains three main parts, namely, (a) Explicitation of PNL propositions in Generalized Constraint Language expressions, (b) Constraint propagation and (c) Retranslation of GCL expressions to NL propositions.

This preliminary analysis of the elements in CTP was based on a detailed review of the following papers by Zadeh:

- 1999 From computing with numbers to computing with words - from manipulation of measurements to manipulation of perceptions [1]

- 2001 A new direction in AI. Towards a Computational Theory of Perceptions [2].

- 2002 Granular computing as a basis for a computational theory of perceptions [3].

The first step consisted of extracting a list of relevant statements representing a preliminary corpus of knowledge about CTP. We performed this conceptual analysis looking for themain concepts and the relation among them. Figures 2 and 3 show two of the concept maps that we elaborated during the exploration of main concepts and their relations in CTP. It is worth noting that, this first work only delivers a provisional result. The analysis of additional literature on CTP will filter out some statements and will add new ones. The preliminary set of obtained statements is listed in the following two subsections:

\subsection{Perceptions as objects of computation}

IDS and TDS in Fig. 1.

- Perceptions are described by propositions $(p)$ drawn from a natural language.

- Perceptions are assumed to be fuzzy and granular.

- A proposition, $p$, is viewed as an answer to a question.

- The meaning of a perception is expressed as a generalized constraint.

- A generalized constraint is represented as $X i s r$ $R$, where $i s r$ is a variable copula that defines the way in which $R$ constrains $X$. This form of representation is called Generalized Constraint Language (GCL).

- PNL is a subset of natural language. It is a set of propositions that have a translation into GCL.

- Translation consists of the explicitation of $X$, $r$, and $R$ from a proposition $(p)$ in PNL.

- In general, $X, r$, and $R$ are implicit rather than explicit in $p$. Furthermore, $X, r$, and $R$ depend on the question to which $p$ is an answer.

- GCL plays the role of a precisiation language, with the understanding that precisiation of meaning is not coextensive with representation of meaning.

- There are concepts that do not admit precisiation within the framework of PNL.

- An explanatory database (ED) consists of a set of perceptions in terms of which the meaning of a perception is defined [8].

- A perceived system $S$ is assumed to be associated with temporal sequences of input, output, and states. $S$ is defined by the statetransition function $f$ and the output function $g$. In perception-based system modeling, the input, the output, the states, $f$ and $g$ are assumed to be perceptions.

- Perception of a function can be described as a collection of linguistic if-then rules, with each rule describing a fuzzy granule.

\subsection{Computing with perceptions}

Process module in Fig. 1.

- Computing with perceptions involves a process of arriving at answers (TDS) to specified questions given a collection of perceptions that constitute the initial data set (IDS). 


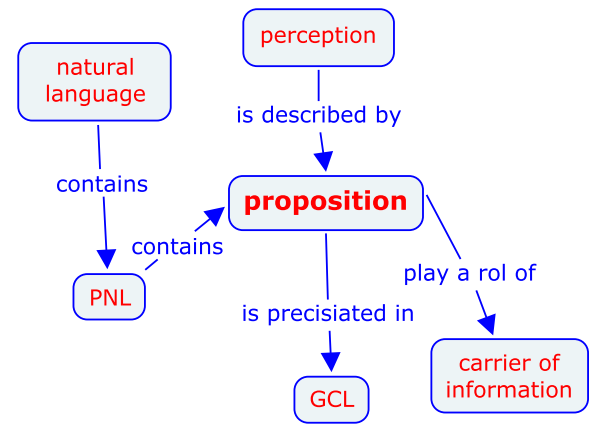

Figure 3: Concept Map of a proposition in CTP

- Computation with perceptions involves a goaldirected propagation of generalized constraints from premises to conclusions.

- General Constraint Propagation (GCP) involves successive application of a collection of rules that govern combination, modification, qualification, and propagation of generalized constraints.

- The principal rule of inference in the computational theory of perceptions is the Generalized Extension Principle.

- The application of the Generalized Extension Principle transforms the problem of reasoning with perceptions into the problem of constrained maximization of the membership function of a variable that is constrained by a query.

- One of the basic rules governing GCP is the compositional rule of inference [9].

\section{Logic of Science}

In this section, we reflect about what type of knowledge about CTP is available in the studied papers. We undertake this analysis using the Logic of Science by K. R. Popper [10].

According to Popper the, so called, rational knowledge, has the formal structure of an axiomatic system. This structure consists of a set of premises and a set of logic rules that allow obtaining the rest of knowledge in a certain domain of knowledge. This axiomatic system must fulfil several formal requirements analyzed in the following subsections.

\subsection{Conditions I}

1. The system of axioms must be free of contradiction (neither self-contradiction nor mutual contradiction)

2. The axioms in the system must be independent, i.e., axioms must not be deducible from the remaining ones.

3. The system of axioms must be sufficient for the deduction of all statements belonging to the domain of knowledge which is to be axiomatized.

4. Every axiom in the system must be necessary, i.e., the system must not contain superfluous assumptions.
In the case of CTP, from our interpretation of the statements in section 2, we have extracted a summary with the form of a set of propositions listed as follows:

1. The kernel of CTP is a computational system with the capacity of computing with the meaning of NL propositions.

2. This computational system is designed to produce linguistic answers to a specific question.

3 . The input to this system is a set of perceptions represented by propositions belonging to a subset of NL called PNL.

4. The meaning of the propositions belonging to PNL can be expressed in GCL, i.e., as fuzzy constraints on the value of a linguistic variable.

5 . The process of obtaining the output from the input is a constraint propagation process.

6. The constraint propagation process can be implemented using the resources provided by Fuzzy Logic.

This list could be considered the starting point for a detailed study aimed to either defining a possible set of axioms for CTP or, more likely, concluding that definition of new concepts is needed to complete an axiomatic system.

\subsection{Conditions II}

According to Popper, an axiomatic system (a theory) should demonstrate its value versus former theories, i.e., the new theory should be contrasted with its rival theories. In this sense, a rational knowledge must contribute to the growth of the available knowledge. This could be done in both directions:

- Providing a higher degree of generality, i.e., the new theory includes the knowledge provided by previous theories.

- Providing a higher degree of precision, i.e., the new theory explains additional details to the knowledge provided by existing theories.

According to Zadeh, CTP is defined on Fuzzy Logic introducing new concepts and possibilities of application [11]. As a demonstration of how CTP can be used to generate new knowledge, in section 6 , following the CTP principia, we introduce a set of definitions that we apply to the development of practical applications.

\subsection{Conditions III}

According to Popper, once these conditions are fulfilled, we could say that the axiomatic system is a Scientific Theory, if it offers statements contrastable with the physical-natural reality.

Perhaps, in the future, CTP will be used to describe a new generation of computational systems with a set of new properties. In its current state of development, we think that CTP can not be called a Scientific Theory in the sense defined by Popper, 
i.e., it does not deal with describing the physicalnatural reality.

\section{Disciplinary matrix}

In this section we reflect about who uses the available knowledge about CTP. We undertake this analysis from the perspective of the disciplinary matrix defined by T.S. Kuhn [12] [13].

According to Kuhn a disciplinary matrix is a model of development of knowledge.

The analysis proposed by Kuhn studies the validity of a theoretical framework in relation with the way the scientific community shares its theoretical, methodological, and technological assumptions.

A specific disciplinary matrix defines the epistemological requirements of a scientific community. From this perspective, we say that the work of a researcher belongs to a certain scientific school when he/she follows a set of theoretical, methodological and technological commitments.

Regarding CTP, we can say that although an important scientific community is working in Fuzzy Logic, the number of researchers in Fuzzy Logic that are interested in CTP is still reduced.

A specific disciplinary matrix contains, namely, symbolic generalizations, models and shared exemplars.

Symbolic generalizations are formalizations that allows to solve problems belonging to the field of knowledge, e.g., Force $=$ Mass $\times$ Acceleration .

Perhaps the most representative symbolic generalization in CTP is the expression of the meaning of a linguistic proposition in GCL.

\section{$X$ isr $R$}

The fundamental model in CTP is the represented in Fig. 1, i.e., a system for obtaining linguistic answers applying a constraints propagation process.

A paradigmatic model in CTP is the model of the meaning of a computational perception using two parallel representations, namely, a linguistic proposition and its expression in GCL.

Shared exemplars are use cases of the considered theory. They define the typical type of problems and solutions belonging to the disciplinary matrix, e.g., the calculus of the gravitational force between the Earth and the Moon.

Zadeh uses typically well known examples to illustrate the principia of CTP. Several of them are the following:

- The example of Mary, Carol and Pat. Here, the query is "How far is Carol from Pat?" [1].

- The example of the Swedes. Here, the query is: "What is the average height of Swedes?" [1].

- The example of Robert. Here, the query is: "What is the probability that Robert is home at about 6:15 pm?" [14].
A disciplinary matrix defines correspondence rules, analogies and metaphors which lead the heuristic research. Additionally it defines the ontological scope of the specific domain of knowledge. E.g., Newton defined the correspondence between the concept of mass and the amount of physical materia of an object. Then he used this correspondence to develop the laws of motion. The analogies in Newton's theory have allowed to extend his proposals to inertial and not inertial physical systems. The well known metaphor of the Newton's apple is still used as motivation, illustration and support for explanations in the domain of the disciplinary matrix.

Regarding CTP, according with the paradigm in AI that consider the computer as a metaphor of mind, we can say that CTP is based on the metaphor of the computational perception as a human perception.

\section{Rationality and Normative theories}

The scientific research deals with increasing human knowledge about the world. Nevertheless, there are domains of knowledge where the available knowledge is used with a normative propose. The normative knowledge is used to perform actions and can be tested either to accept or reject its validity, i.e., this type of knowledge is devoted to achieve practical goals. E.g. the rules for playing golf are a normative knowledge.

According with Hansson, a normative theory is a theory about how decisions should be made. Moreover, a normative theory is a theory about how decisions should be made in order to be rational [15].

A normative theory must fulfil the following requirements:

- It aims at practical decisions

- It is applicable to real cases

- Its proposals are viable

- It is suitable to achieve its goals

According with the references, CTP could be interpreted as a practical framework to develop computational systems with the capacity of computing with the meaning of NL expressions. Of course, this potentiality should be demonstrated with successful applications.

CTP is intended to be applicable to real world problems. "When the available information is too imprecise to justify the use of numbers; and second, when there is a tolerance for imprecision which can be exploited to achieve tractability, robustness, low solution cost and better rapport with reality. Exploitation of the tolerance for imprecision is an issue of central importance in CW and CTP" [1] (pg. 2). A positive argument on the applicability of CTP is supported by the impressive practical applications of Fuzzy Logic. 
A methodology for development of practical projects based on CTP would increase its applicability and is needed to demonstrate its normative value.

\section{Towards applications of CTP}

In the remainder of this paper, we contribute to the development of the practical aspects of CTP by providing a set of definitions aimed to establish a framework for developing applications.

These definitions are a result of our work developing computational applications based on the fundamentals of CTP. Our current research, is focused on developing computational systems able to provide users with meaningful linguistic descriptions of the phenomena in their environment [16] [17] [18] [19] [20] [21] [22]. During these works, we have needed to answer a list of questions that we answer in the following sections:

- Which is the object of perceptions?

- Who is subject of perceptions?

- Who is the reader/listener of the NL propositions?

- What is a computational perception?

- How to model the granularity of perceptions and the constraints propagation process?

\subsection{The object of perceptions. Phenomena}

According to Zadeh, the object of perceptions are not only the attributes of objects, e.g., the distance, velocity and angle. The object of perception can be the whole systems, e.g., a person parking a car and the traffic in a roundabout.

We use the term phenomenon to represent a system that is perceived while it is evolving in different situation types.

We have taken this term from Systemic Functional Linguistic (SFL), where phenomena are defined in terms of participants, process and circumstances according with the NL structure [23] [24].

\subsection{The subject of perceptions. The designer}

The computational model of a phenomenon is based on subjective perceptions of a domain expert that we call the designer. The more experienced designer, with better understanding and use of NL, the richer the model with more possibilities of achieving and responding to final users' needs and expectations.

The designer uses the resources of the computer, e.g., sensors, to acquire data about a phenomenon and uses his/her own experience to interpret these data and to create a model of the phenomenon. Then the designer uses the resources of the computer to produce the linguistic utterances. Moreover, we can say that the designer is who speaks when the computer speaks.
The designer uses NL with two functions, namely, to represent his/her experience and to communicate with others [23]. The designer uses the first function to build the linguistic model of a phenomenon and uses the second function to communicate information about this phenomenon to the users of the computational system.

\subsection{Reader/listener of the NL propositions}

During the development phase of the project, readers of the linguistic model of a phenomenon are the own designer and his/her working team. We can say that they use this type of model as a software engineering tool to represent their interpretation of the perceived phenomenon.

During the exploitation phase of the project, come computational applications of CTP produce NL utterances, i.e., they communicate with people using NL. The users of a specific application have specific experience and personal use of NL. Moreover, they have specific goals in the particular context of the application. The model must be designed taking into account all these aspects.

\subsection{Computational perception (CP)}

$\mathrm{CP}$ is the computational model of a unit of information acquired by the designer about the phenomenon to be modeled. $\mathrm{CP}$ is a couple $(A, w)$ where:

$A$ is a NL sentence. This sentence can be either simple, e.g., $A=$ "The temperature is high" or more complex, e.g., $A=$ "The efficiency of energy consumption has decreased during the last semester".

$w \in[0,1]$ is the degree of validity of $A$ in a specific context. The concept of validity depends on the application, e.g., it is function of the truthfulness and relevancy of the sentence in its context of use.

\subsection{Computational perception protoform (CPP)}

The concept of protoform was introduced by Zadeh as "[...] a symbolic expression which defines the deep semantic structure of an object such as a proposition, question, command, concept, scenario, case, or a system of such objects" [25]. Here, $\mathrm{CPP}$ is a generalization of $\mathrm{CP}$ that represents the whole possible values of $\mathrm{CP}$, i.e., $\left(A_{i}, W_{i}\right)=$ $\left\{\left(A_{1}, w_{1}\right),\left(A_{2}, w_{2}\right), \ldots,\left(A_{n}, w_{n}\right)\right\}$. CPP is used to represent the possibilities of evolution of a specific phenomenon, in a specific situation type, and with a certain degree of granularity.

For example, the set of possible values of CP representing the temperature in a room could be represented as follows: 


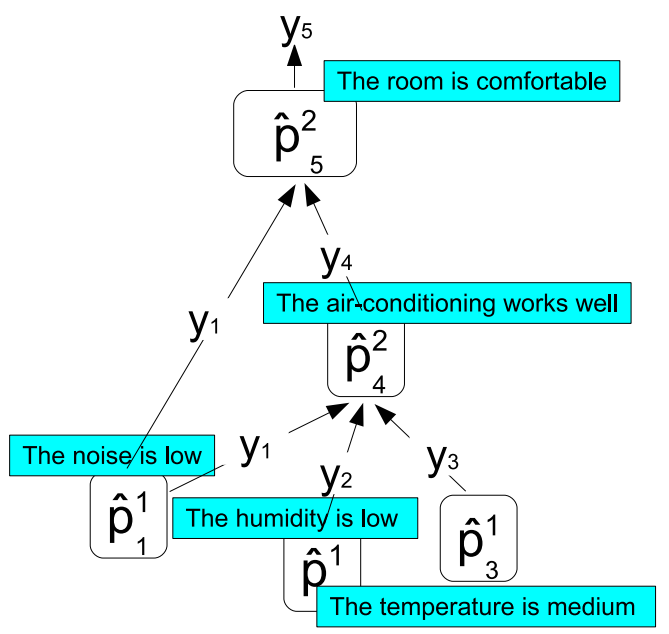

Figure 4: Simple example of GLMP.

$\left\{\left(\right.\right.$ The temperature in the room is high, $\left.w_{1}{ }^{\prime \prime}\right)$,

(The temperature in the room is medium, $w_{2}$ ),

(The temperature in the room is low, $w_{3}$ ) \}

\subsection{Perception mapping}

We call perception mapping (PM) to a function that performs the constraint propagation process, i.e., the function that converts the input perceptions in output perception (see Fig. 1). We call perception mapping protoform (PMP) to a generalization of $\mathrm{PM}$, i.e., PMP is a generic function that allow to obtain a type of TDS from certain types of IDS.

\subsection{First-order perception mapping protoforms (1-PMP)}

The designer uses 1-PMP to define the maximum level of detail (the highest granularity) in the linguistic model of a phenomenon. 1-PMPs correspond with the designer's interpretation of input data $(u)$, e.g., sensor data. 1-PMP is a tuple $(u, y, g, T)$ where:

$u$ is a variable defined in the input data domain, e.g., the value $u \in \mathcal{R}$ provided by a thermometer.

$y$ is an output CPP, e.g., The temperature in a room. It contains values $y=\left(A_{y}, W_{y}\right)=$ $\left\{\left(A_{1}, w_{1}\right),\left(A_{2}, w_{2}\right), \ldots,\left(A_{n_{y}}, w_{n_{y}}\right)\right\}$.

$g$ is a set of membership functions

$$
\begin{gathered}
W_{y}=\left(w_{1}, w_{2}, \ldots, w_{n_{y}}\right)=g(u)= \\
\left(\mu_{A_{1}}(u), \mu_{A_{2}}(u), \ldots, \mu_{A_{n_{y}}}(u)\right)
\end{gathered}
$$

where $W_{y}$ is the vector of degrees of validity assigned to each $A_{y}$ and $u$ is the input data.

$T$ Here, it is typically a simple template that allows generating the elements in $A_{y}$, e.g., "The temperature in the room is $\{$ high $\mid$ medium $\mid$ low $\}$ ".
The designer uses 1-PMPs to define a mapping between the numerical domain of input data and a domain of linguistic expressions. We say that the output of a 1-PMP is based on information taken outside of the model, i.e., in the context of the model. Note that, apart of sensors data, the designer can define first-order computational perceptions (1-CP) from data of different sources, e.g., a table of data from Internet.

\subsection{Second-order perception mapping protoform (2-PMP)}

Perceptions whose meaning is based on other subordinate perceptions are called second-order perceptions (2-CP). 2-CPs are outputs 2-PMP.

A 2-PMP is a tuple $(U, y, g, T)$ where:

$U$ is a set of input CPP $\left(u_{1}, u_{2}, \ldots, u_{n}\right)$.

$y$ is the output $\mathrm{CPP}$ with values $y=\left(A_{i}, W_{i}\right)=$ $\left\{\left(A_{1}, w_{1}\right),\left(A_{2}, w_{2}\right), \ldots,\left(A_{n_{y}}, w_{n_{y}}\right)\right\}$.

$g$ is the aggregation function.

$$
W_{y}=g\left(W_{u_{1}}, W_{u_{2}}, \ldots, W_{u_{n}}\right)
$$

where $W_{y}$ is a vector $\left(w_{1}, w_{2}, \ldots, w_{n_{y}}\right)$ of degrees of validity assigned to each element in $y$ and $W_{u_{i}}$ are the degrees of validity of the input perceptions. The designer chooses the most adequate aggregation function to each case. In Fuzzy Logic many different types of aggregation functions have been developed. Typically $\mathrm{g}$ is implemented using a set of fuzzy rules.

$T$ is a text generation algorithm that allows generating the sentences in $A_{y}$. Our research in this algorithm is currently focused on Systemic Functional Linguistic.

\subsection{Granular Linguistic Model of a Phenomenon (GLMP)}

In each situation type, the designer uses a network of PMPs to create a description of the monitored physical phenomenon with different levels of granularity that we call GLMP.

Each PMP receives a set of input CPs from its subordinate PMPs and transmits upwards the output CP. We say that each output CP is explained by a set of input CPs.

In the network, each PMP covers specific aspects of the phenomenon with certain degree of granularity. Each PMP has associated a set of linguistic clauses, each one with an associated degree of validity, that covers the whole of occurrences of the phenomenon from the designer's perspective.

Figure 4 shows an example where three 1-PMP $\hat{p}_{1}^{1}, \hat{p}_{2}^{1}, \hat{p}_{3}^{1}$ are used to explain a 2-PMP $\hat{p}_{4}^{2}$. Then $\hat{p}_{1}^{1}$ and $\hat{p}_{4}^{2}$ explain $\hat{p}_{5}^{2}$. Here $\hat{p}_{5}^{2}$ is called the top-order perception, i.e., a perception that answers a general question about the phenomenon. 
Using different aggregation functions and different linguistic expressions, the paradigm GLMP provides the designer with a useful tool to model his/her perceptions according to his/her own experience and use of NL.

\subsection{Draft methodology}

In our approach, a basic development methodology of applications can be summarized as follows:

1. Study the application domain and elaborate a corpus of typical linguistic expressions used in the area.

2. Use SFL to create an ordered structure that initially can be viewed as a collection of possible questions and their possible answers (use cases). Each question is associated to a tree of choices that permits selecting the most adequate response in each situation.

3. Create a GLMP and use the resources provided by Fuzzy Logic, assigning to each possible response a validity degree in the different situation types and depending on the input data.

4. Create a constraints propagation process able to select among the possible options the most relevant response for the user depending on the context defined by the input data.

\section{Conclusions}

This is a very preliminary work on the epistemology of CTP that provide several provisional conclusions.

From the perspective of the Logic of Science, we have used a representative corpus of information about CTP to explore the existence of a possible axiomatic system that fulfills the requirements of rational knowledge. The preliminary conclusion is that currently, CTP has not a definition based on an axiomatic system.

Using the definition by Kuhn, we have seen that CTP contains partially the needed elements to be considered a disciplinary matrix.

Finally, we conclude that essentially CTP is aimed to be a future normative theory, i.e., after additional development, CTP could provide a practical framework to develop a new type of computational applications. In order to support this conclusion, following the CTP fundamentals, we have presented some practical definitions that could be useful for the development of CTP as a normative theory.

\section{Acknowledgment}

This work has been funded by the Foundation for the Advancement of Soft Computing (Mieres, Asturias, Spain), by the Spanish Government (CICYT) under project TIN2008-06890-C02-01 and by the Canary Islands Government under scholarship BOC119-18-06-2010.

\section{References}

[1] L. A. Zadeh. From computing with numbers to computing with words - from manipulation of measurements to manipulation of perceptions. IEEE Transactions on Circuits and Systems, 45(1), 1999.

[2] L. A. Zadeh. A new direction in AI. towards a computational theory of perceptions of measurements to manipulation of perceptions. AI Magazine, 22(1), 2001.

[3] L. A. Zadeh. Granular computing as a basis for a computational theory of perceptions. In Proceedings of the IEEE International Conference on Fuzzy Systems, pages 564-565, Honolulu, USA, May 2002.

[4] L. A. Zadeh. Toward human level machine intelligence - is it achievable? the need for a paradigm shift. IEEE Computational Intelligence Magazine, August 2008.

[5] L. A. Zadeh. Fuzzy sets and information granularity. Advances in Fuzzy Set Theory and Applications, pages 69-129, 1979.

[6] L. A. Zadeh. Towards a theory of fuzzy information granulation and its centrality in human reasoning and fuzzy logic. Fuzzy Sets and Systems, 90:111-127, 1997.

[7] L. A. Zadeh. The concept of linguistic variable and its application to approximate reasoning. Information sciences, 8:199-249, 1975.

[8] L. A. Zadeh. Outline of a computational approach to meaning and knowledge representation based on the concept of a generalized assignment statement. In Proceedings of the International Seminar on Artificial Intelligence and Man-Machine Systems, pages 198-211, 1986.

[9] L. A. Zadeh. Outline of a new approach to the analysis of complex systems and decision processes. IEEE Transactions on Systems, Man and Cybernetics, smc-3, 1, January 1973.

[10] K.R. Popper. The Logic of Scientific Discovery. Hutchinson \& Co., London, 1959.

[11] Lotfi A. Zadeh. Fuzzy logic = computing with words. IEEE Transactions on Fuzzy Systems, 4:103-110, 1996.

[12] T. S. Kuhn. The Structure of Scientific Revolutions. University of Chicago Press, 1970.

[13] T. S. Kuhn. Second Thoughts on Paradigms: The Structure of Scientific Theories. Urbana University of Illinois Press, 1974.

[14] L. A. Zadeh. From search engines to question answering systems. the problems of world knowledge, relevance, deduction and precisiation. In E. Sanchez, editor, Fuzzy Logic and the Semantic Web. Elsevier, Amsterdam, The Netherlands, 2006.

[15] S.O. Hansson. Decision theory. a brief introduction (2005).

[16] S. Mendez-Nunez and G. Trivino. Combining 
semantic web technologies and computational theory of perceptions for text generation in financial analysis. In Proceedings of the IEEE Fuzzy 2010, Barcelona, Spain, 2010.

[17] G. Trivino, A. Sanchez, A. S. Montemayor, J. J. Pantrigo, Raul Cabido, and E. G. Pardo. Linguistic description of traffic in a roundabout. In Proceedings of the IEEE Fuzzy 2010, Barcelona, Spain, 2010.

[18] G. Trivino, A. Alvarez, and G. Bailador. Application of the computational theory of perceptions to human gait pattern recognition. Pattern Recognition, 43-7:2572-2581, 2010.

[19] G. Trivino and A. Sobrino. Human perceptions versus computational perceptions in computational theory of perceptions. In Proceedings of the International Fuzzy Systems Association World Congress, IFSA/EUSFLAT, Lisboa, Portugal, July 2009.

[20] G. Trivino and A. van der Heide. An experiment on the description of sequences of fuzzy perceptions. In Proceedings of the 8th International Conference on Hybrid Intelligent Systems (HIS2008), Barcelona, Spain, September 2008.

[21] L. Eciolaza and G. Trivino. Fuzzy linguistic reporting in driving simulators. In Proceedings of the IEEE Symposium Series on Computational Intelligence (SSCI 2011), Paris, France, 2011.

[22] C. Menendez and G. Trivino. Olap navigation in the granular linguistic model of a phenomenon. In Proceedings of the IEEE Symposium Series on Computational Intelligence (SSCI 2011), Paris, France, 2011.

[23] M. A. K. Halliday and M. I. M. Matthiessen. Construing Experience through Meaning: A Language-based Approach to Cognition. Continuum; Study ed edition (June 3, 2006), 1999.

[24] M. A. K. Halliday and M. I. M Matthiessen. An Introduction to Functional Grammar. Oxford University, New York, 2004.

[25] L. A. Zadeh. Toward a generalized theory of uncertainty (GTU). An outline. Information Sciences, 172:1-40, 2005. 